\title{
Socioéconomie des oléagineux en Afrique
}

\author{
Antoine LABEY \\ 23 Grand Rue, 34920 Le Cres, France \\ <alabey@wanadoo.fr>
}

\begin{abstract}
Africa lost, over the years, its dominant rank on the international market of oilseeds, enhancing some asian giants such as Indonesia and Malesia which have been establishing large industrial plantations since the seventies. This must not lead to ignore the fact that, in African countries which produce them, oilseeds play a paramount socio-economic role. Oil plants are, most of the time, cultivated in family farms and participate in the survival, thus the maintenance, of the farmers on their croplands. In some cases, they are the only source of cash income in an agricultural system of almost selfsubsistence. In recent years, cultivation of oilseeds has become a more profitable activity, unlike coffee and cocoa whose prices keeps decreasing. Therefore, the renewed interest of farmers to them is consequent, particularly to the most important of them, which is the oil palm, mainly grown in Cameroon, Ivory Coast and Nigeria. Saturation of croplands in Asia gives real opportunities of development in Africa, provided that the environmental issue must be considered (deforestation, monoculture...). Cottonseed oil, rather unknown in Europa, also becomes very important because of its cheapness and its high rate of consumption as edible oil in producing countries. Groundnut's situation is more concerning: a hard crisis hits thousands of Senegalese farmers in areas of high poverty. Shea butter is special as a product because it is not cultivable. It is harvested only on wild trees with all the provided uncertainties. The demand for this product is strong in the industrialised countries because of its cosmetic features. Finally, Africa is also exploited for the production of biodiesels. The issue is complicated and, after a first period of craze, some countries start to reduce their claims in this field. Demand for oils keeps increasing: will Africa be able to benefit from that strongly increased demand, and under what conditions? Organisation of chain, investment, competitiveness, access to the markets... are all determining features.
\end{abstract}

Key words: Africa, oilseeds, palmoil, cottonseeds, groundnut, shea butter, biodiesels
En Afrique, la culture des oléagineux a suivi la même logique coloniale que les autres grandes spéculations agricoles (café, cacao, coton...) : celle qui consistait à subvenir aux besoins de la métropole en produits qu'elle ne pouvait pas produire principalement pour cause d'incompatibilité géoclimatique. Pourtant, durant ces vingt ou trente dernières années, le continent africain est devenu un acteur marginal sur le marché mondial des oléagineux suite au déclin de sa production et de ses exportations. De nouveaux acteurs, plus performants, se sont imposés sur le marché au détriment de l'Afrique. Le soja s'est imposé, propulsé par l'augmentation exponentielle de la demande en produits pour l'alimentation animale et avec lui ses principaux producteurs: ÉtatsUnis, Brésil, Chine, Argentine... où la production a littéralement explosé dans les années 1990. Quant à l'huile de palme, elle a vu la montée en puissance des producteurs asiatiques qui sont venus détrôner l'Afrique. Aujourd'hui, après avoir approvisionné l'Europe durant des décennies, I'Afrique occupe une place marginale sur le marché mondial. Elle est même importatrice de certaines huiles.
Si on s'en tient à l'Afrique francophone, qui s'étend sur toute l'Afrique de l'ouest et du centre, du Sénégal au nord jusqu'au Congo au sud, on distingue deux grandes zones de production liées à deux grands ensembles climatiques: le Sahel et I'Afrique tropicale. Le Sahel est la région de l'arachide, surtout le Sénégal et le nord du Nigeria, du coton et du karité. La zone tropicale abrite les cultures de palmier à huile avec des plantations tout le long des côtes du golfe de Guinée depuis la Sierra Leone, le Liberia et la Côte-d'Ivoire jusqu'au Cameroun et au Gabon en passant par le Nigeria. Le palmier à huile y côtoie le café, le cacao et bien sûr les grandes exploitations forestières. Le palmier à huile est d'ailleurs originaire de cette région du Golfe de Guinée où il existait à l'état naturel et est exploité depuis la nuit des temps.

\section{Déclin de l'huile de palme}

L'huile de palme est la plus importante culture oléagineuse en termes de volumes. L'Afrique était un gros producteur d'huile de palme au moment des indépendances, au début des années soixante, mais la production a pratiquement stagné au cours de ces dernières décennies tandis que l'Asie se lançait dans des vastes projets de plantations industrielles. Aujourd'hui, I'Indonésie et la Malaisie dominent totalement le marché mondial de l'huile de palme. En 1960, I'Afrique réalisait $99 \%$ des exportations d'huile de palme et I'Indonésie et la Malaisie $1 \%$ à elles deux. Aujourd'hui, la part de ces deux pays est de $80 \%$. Mais la montée en puissance de ces deux pays s'est faite au prix d'un désastre écologique de grande ampleur avec la destruction de centaines de milliers $d^{\prime}$ 'ha de forêts primaires, l'expulsion des populations indigènes qui y vivaient et la disparition d'un grand nombre d'espèces animales et végétales. Certains industriels tentent encore, contre toute évidence, de minimiser les dégâts causés par cette monoculture industrielle à grande échelle.

L'Afrique a heureusement échappé à ce destin. À l'opposé des systèmes asiatiques basés sur des grandes exploitations industrielles gérées par des grands groupes, l'Afrique a préservé un mode de culture familial qui, s'il ne permet 
pas d'atteindre les rendements obtenus dans les grandes exploitations asiatiques, a permis de conserver le tissu socio-économique et l'équilibre écologique de la région. C'est là I'une des causes fondamentales de la perte de vitesse du continent africain sur le marché international. Au Cameroun par exemple, la grande majorité des exploitations de palmier à huile (7 sur 10) font moins de 3 ha. En Asie, les plantations industrielles qui assurent $80 \%$ de la production mondiale mesurent entre 2500 et 10000 ha.

Le manque de compétitivité des palmeraies africaines s'explique également par des conditions bioclimatiques : les mêmes plants cultivés en Asie et en Afrique dans des conditions expérimentales similaires donnent des rendements très différents. En outre, les exploitations familiales africaines ne bénéficient pas des résultats de la recherche sur les variétés, la culture et même l'extraction. Au bout du compte, la productivité atteint en moyenne $2 \mathrm{t} /$ ha en Afrique contre 3,5 tonnes en Indonésie et en Malaisie. Les politiques économiques suivis par les États africains depuis l'indépendance expliquent également le déclin de la production africaine $\mathrm{d}^{\prime}$ huile de palme. Au début des indépendances, des grandes sociétés d'État ont été mises en place pour succéder au système d'exploitation colonial. Chargées d'encadrer les petits producteurs, elles ont très imparfaitement rempli leur rôle. Lourdement gérées par une administration pléthorique, budgétivores et mal orientées, sans politique clairement définie, elles se sont révélées totalement inadaptées à leur fonction. Au milieu des années soixantedix, le problème de la dette et les mesures d'ajustement structurel imposées par la Banque mondiale et le Fonds monétaire international qui en ont résulté, ont conduit à la dissolution plus ou moins expéditive de ces sociétés d'État devenues une proie facile de la rationalisation économique. Les planteurs se sont alors retrouvés livrés à eux-mêmes, privés des services que leur rendaient malgré tout ces sociétés d'État au niveau de la fourniture des intrants, de la commercialisation de la production et de la vulgarisation.

La richesse et l'intérêt de la palmeraie africaine tiennent en grande partie à sa forte intégration dans l'exploitation familiale. Elle participe à la diversification des cultures pour éviter les risques économiques de la dépendance à une monoculture où une mauvaise récolte met en jeu la survie de l'exploitation et de ceux qui vivent dessus. Le palmier à huile est souvent cultivé avec l'hévéa et les cultures vivrières. La palmeraie est souvent la seule source de revenus monétaires dans des exploitations qui restent tournées vers l'autosubsistance.

En dehors de l'huile, la palmeraie fournit de nombreux autres produits. Les sous-produits issus de l'extraction artisanale telle qu'elle est pratiquée en Afrique sont très utilisés. Les fibres sont brûlées dans les chaudières pour produire de l'électricité pour I'huilerie et pour les villages environnants. Les rafles sont utilisées comme compost dans les champs. Les tourteaux servent à I'alimentation du bétail, petit et gros. On fait même de l'alcool appelé «vin de palme », fabriqué en brousse dans des distilleries artisanales plus ou moins clandestines mais généralement tolérées (cette boisson fortement alcoolisée peut avoir des effets redoutables pour les non-initiés).

\section{Un nouveau leader régional}

Aujourd'hui, après avoir été un exportateur de premier plan, I'Afrique est confrontée au défi de redevenir au moins autosuffisante en huile de palme qui est, de loin, la principale huile alimentaire consommée sur le continent.

Pour une consommation d'environ 1,3 millions de tonnes sur l'ensemble de l'Afrique de l'ouest (zone Cedeao), il manque environ 200000 à 300000 tonnes qui sont couvertes par les importations en provenance d'Asie. La production locale provient surtout du Nigeria (800 000 tonnes) et de la Côte-d'Ivoire (300 000 tonnes). Cette situation n'a pas échappé à deux puissants groupes industriels de Singapour, Olam et Wilmar, qui ont signé fin 2007 un important contrat avec la Sifca, la première entreprise agro-industrielle ivoirienne très présente dans le palmier à huile, l'hévéa et le sucre. L'objectif est clair : combler ce déficit actuel en huiles de l'Afrique de l'ouest et répondre à la rapide augmentation de la demande. Les investissements prévus sont à la hauteur de l'enjeu : au moins 200 millions de dollars en nouvelles plantations et usines de traitement en Côte d'Ivoire et dans les pays voisins.

Olam est un très important négociant en matières premières agricoles, très présent en Afrique où il achète une vaste gamme de produits tropicaux pour ses clients asiatiques. Wilmar a été fondée en 1991 en tant que négociant en huile de palme. Ses activités se sont depuis élargies à l'amont de la filière depuis la culture du palmier jusqu'à l'industrie de transformation. C'est aujourd'hui le plus gros transformateur d'huile de palme dans le monde. Quant à la Sifca, basée en Côte-d'Ivoire, c'est une holding qui regroupe trois sociétés de production de caoutchouc en Côte-d'Ivoire (SAPH), au Ghana (Grel) et au Nigéria (MDC), deux sociétés de production (Palm $\mathrm{Cl}$ ) et de raffinage (Cosmivoire) d'huile de palme en Côte-d'Ivoire et d'huile de coton au Bénin (SHB) et une société de production de sucre de canne en Côte-d'Ivoire (Sucrivoire). Palm $\mathrm{Ci}$, que l'association va désormais contrôler grâce à des apports de capitaux des partenaires asiatiques est leader régional avec $80 \%$ du marché ivoirien et $75 \%$ du marché de I'UEMOA pour I'huile de palme brute. Newco, que le groupement va racheter à Unilever, détient $30 \%$ de ces mêmes marchés pour I'huile raffinée. Pour les trois compagnies concernées: Olam, Wilmar et Sifca, cette alliance stratégique tripartite doit leur permettre de développer une position de leadership au niveau régional dans I'huile de palme, le caoutchouc naturel, le sucre et potentiellement dans $d^{\prime}$ autres cultures tropicales. Wilmar apportera son expertise technique, Olam sa connaissance du marché et son réseau de distribution en Afrique de l'ouest. Quant à Sifca, elle apporte son implantation en Côte-d'Ivoire, un marché difficile à pénétrer sans relais local.

Cette alliance, importante par sa dimension et les investissements qu'elle met en jeu, l'est aussi d'un point de vue stratégique. C'est en effet la première fois qu'un groupe asiatique décide de s'implanter directement et d'investir sur le marché africain pour l'approvisionner. Olam et Wilmar prennent ainsi la place laissée vacante par les sociétés d'État qui encadraient les planteurs jusqu'à leur dissolution et par les sociétés occidentales surtout françaises et belges, qui se sont progressivement retirées. Elles viennent aussi profiter des terres disponibles en Afrique de l'ouest pour des nouvelles plantations alors que l'Asie a atteint le stade de saturation pour les plantations compte tenu de l'extrême pression foncière.

\section{Coton, arachide et karité...}

Une autre huile beaucoup moins connue que I'huile de palme est l'huile de coton. L'usage de cette huile est pourtant très répandu dans les zones de production cotonnière d'Afrique de l'ouest qui disposent de bonnes huileries pour traiter ce sous-produit issu de l'égrenage du coton. L'huile de coton y représente la première source de matière grasse utilisée (sous forme d'huile ou transformée en margarine). Sa forte proportion en acide linoléique lui confère de vertus nutritionnelles importantes L'huile de coton est également employée dans la fabrication de savons et de cosmétiques. L'huile de coton est extraite des graines par trituration (figure 1). Ces graines contiennent $18 \%$ d'huile. Environ les deux tiers des graines de coton sont destinées à la production d'huile alimentaire. Selon les données du Département américain de l'agriculture de décembre 2008, la production mondiale d'huile de coton se serait classée au cinquième rang des huiles végétales produites en termes de volume sur la campagne 2007-2008 avec un peu moins de $4 \%$ de la production mondiale.

La situation de l'arachide est beaucoup plus problématique et incertaine (figure 2). Le bassin 


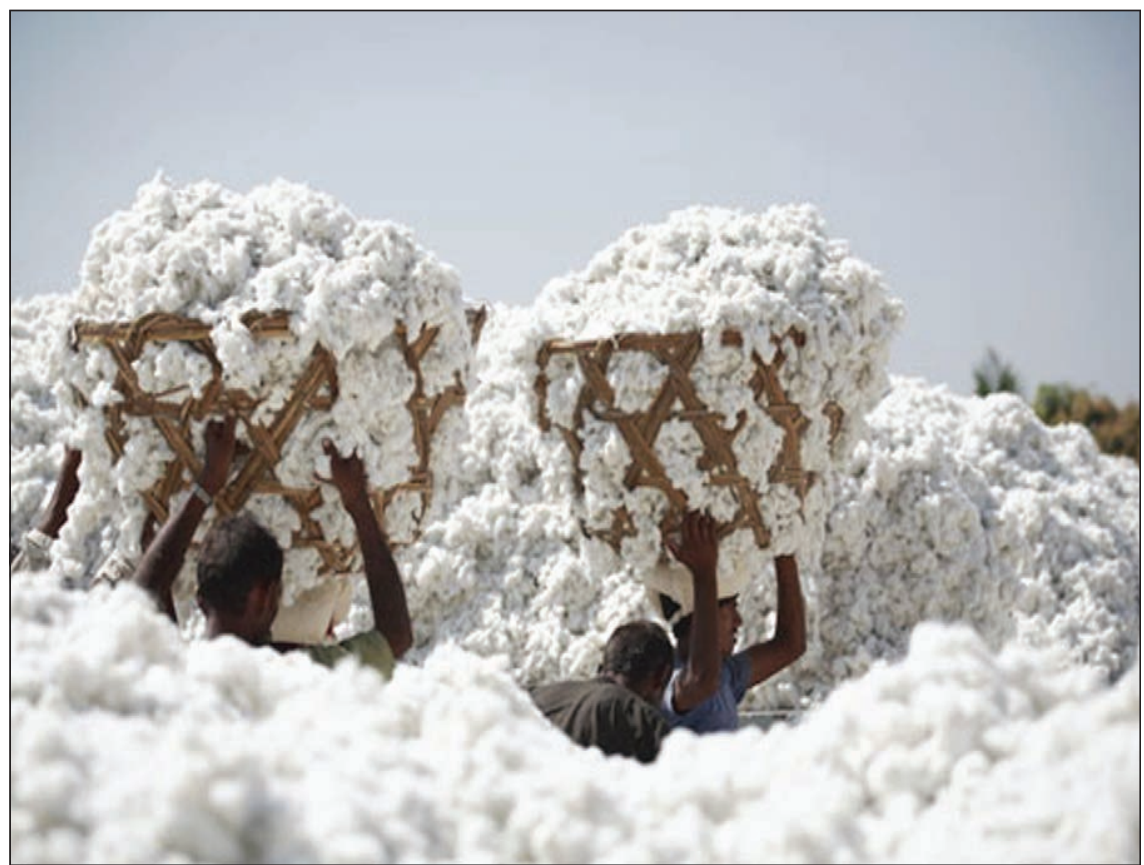

Figure 1. Au-delà de la fibre, le coton sous forme d'huile et de tourteau représente une contribution importante des besoins locaux.

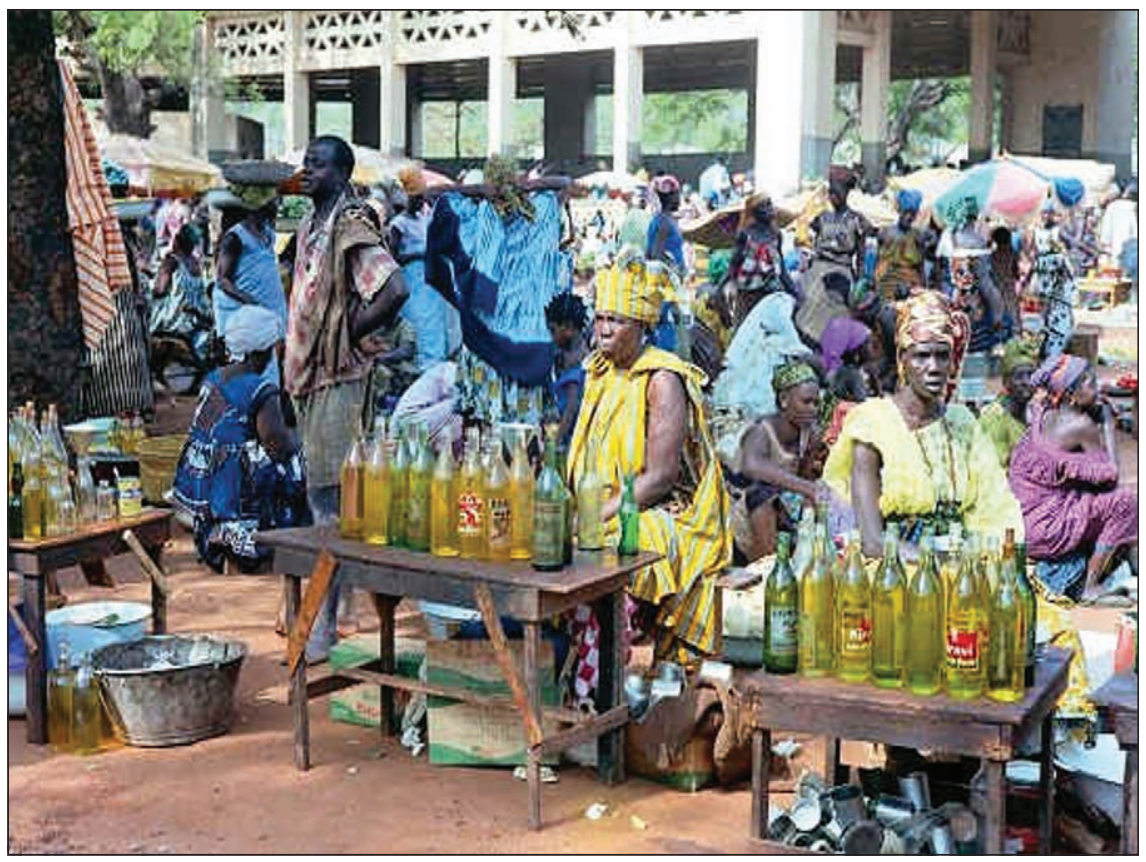

Figure 2. Sénégal, marché. arachidier sénégalais qui s'étend sur tout I'ouest du pays de Saint-Louis, au nord, à la limite de la Casamance, au sud, traverse une crise extrêmement grave et profonde et cela fait des années que cela dure, sans qu'une solution semble en vue. L'État a longtemps soutenu à bout de bras les quatre millions de producteurs en subventionnant à la fois les intrants et tains se demandent pourquoi Advens est venu se mettre dans ce bourbier de l'arachide sénégalaise. À ceux-là, d'autres font remarquer qu'en contrepartie de la reprise en main de la filière arachidière, l'État sénégalais a offert des avantages importants à Advens, qui est avant tout un groupe de négoce d'huiles. II a notamment obtenu une situation de quasi-monopole sur le marché des huiles sénégalaises.

Quant au karité, c'est un produit très singulier à bien des égards. On ne peut pas évoquer les oléagineux africains sans parler de ce produit magique. Magique d'abord parce que le karité ne se laisse pas domestiquer. En effet, l'amande de karité dont on tire le précieux beurre de karité provient du fruit d'un arbre, le karité, qui pousse dans les régions sahéliennes et de savanes depuis le Sahel jusqu'au Cameroun.

$C^{\prime}$ est un arbre robuste de 15 à 20 mètres de haut. Jusqu'à présent, on n'a pas réussi à le reproduire pour le cultiver. Dans tous les cas, il est à croissance très lente comme tous les arbres qui résistent à des climats extrêmes et il ne commence à produire qu'à partir de 20 ans pour atteindre un rythme maximum à 35 40 ans. D'où les problèmes que poserait sa culture, en termes d'investissements. II s'agit donc d'une économie de cueillette traditionnellement effectuée par les femmes qui assurent ensuite le broyage des amandes. Dans ce sens, l'économie du karité joue un rôle socioéconomique très important car il procure un revenu monétaire aux femmes qui le cueillent et le transforment, alors qu'au Sahel, ce sont généralement les hommes qui s'occupent des cultures destinées à la vente : coton et céréales. Les revenus tirés du maraîchage qui est du ressort des femmes sont aléatoires et modestes.

Dans certaines régions où il pousse, le karité revêt un caractère sacré. C'est un arbre vénéré qui est très rarement abattu. Le karité est comestible. II en a été beaucoup question au moment où l'Union européenne a décidé $d$ 'autoriser d'autres graisses que le beurre de cacao dans la fabrication du chocolat; une affaire qui a fait grand bruit et a suscité une levée de bouclier de la part des producteurs de cacao et des puristes du chocolat qui voulaient lui garder son authenticité. On a alors évoqué le beurre de karité comme substitut possible au beurre de cacao. En fait, c'était créer un faux espoir dans la mesure où les fabricants de chocolat se procurent plus facilement de I'huile de palme, de surcroît moins chère. Le fait que le karité ne soit pas cultivé fait qu'il n'y a pas de garantie de régularité et de qualité des approvisionnements, ce dont se plaignent souvent certains négociants occidentaux. La filière ne s'est structurée que récemment, souvent avec le soutien de petits projets de développement qui ont mis en place des groupements de producteurs(trices)-transformateurs la récolte. La gestion de la filière a finalement été privatisée et attribuée au groupe français Advens. Les problèmes de la filière arachidière sénégalaise sont complexes et profonds : problème de financement des campagnes, retard de paiement des achats, débouchés de plus en plus incertains avec une marginalisation de I'huile d'arachide sur le marché mondial. Cer- 


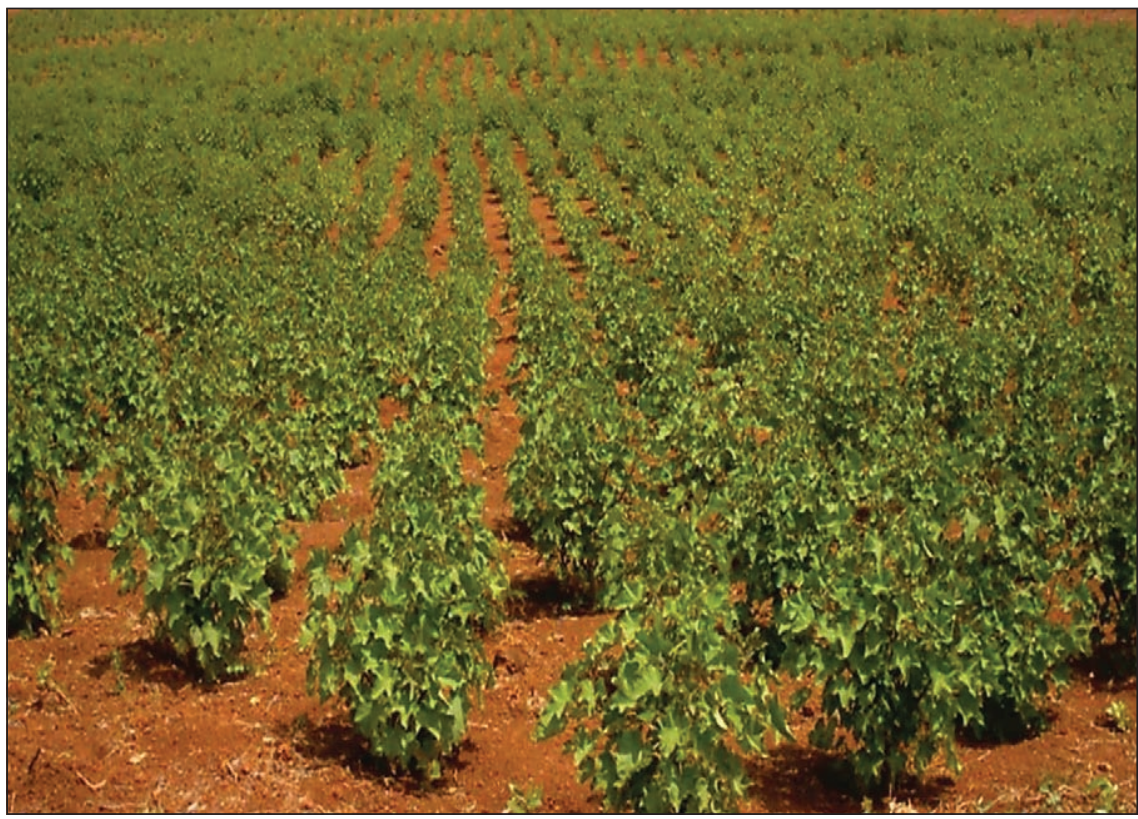

Figure 3. Plantation de jatropha.

(trices) afin de permettre aux acheteurs-grossistes d'avoir un interlocuteur bien défini. Mais le système n'est pas encore totalement opérant. En dehors de ses propriétés nutritionnelles, le karité est surtout connu dans le domaine des cosmétiques. C'est même un produit à la mode et tant mieux car il a de réelles propriétés bienfaitrices pour la peau.

\section{Le jatropha, culture d'avenir?}

Quant au jatropha, il en est beaucoup question depuis que l'on parle des agrocarburants (figure 3). Le jatropha est en effet parti prenante nourrir la planète commencent à être une préoccupation majeure. Dès lors, certains s'interrogent sur l'opportunité pour l'Afrique de se lancer dans cette aventure des agrocarburants en se consacrant à des nouvelles cultures d'exportation alors qu'elle devrait avant tout chercher à répondre à ses propres besoins alimentaires plutôt que céder ses terres agricoles pour satisfaire les besoins du Nord (figurer 3). Les défenseurs du jatropha soulignent que cette plante peut se contenter de terres pauvres, impropres à la culture céréalières, donc qu'elles n'entrent pas en concurrence avec les cultures alimentaires. Mais il n'y a pas de magie : pour obtenir des rendements intéressants et donc en faire une culture rentable, il lui faut une bonne terre. Dès lors, les investisseurs peuvent être tentés de louer ou d'acheter des bonnes terres pour cela au détriment des cultures vivrières. Cette question rejoint le débat actuel sur le vaste mouvement de cession des terres en Afrique à des investisseurs étrangers dont certaines sont destinées à la culture d'agrocarburants. D'autres, plus pragmatiques comme la géographe Sylvie Brunel, estime («Le Monde » du 30 juin 2009) que « la question essentielle n'est pas tant le type de cultures qu'il faut pratiquer - vivrière, industrielle, biocarburants, exportations - que la garantie de revenu qu'elle apporte au paysan ».

Alors que la demande d'huile explose au niveau mondial, aussi bien pour les usages alimentaires que pour les agrocarburants, la question est posée de savoir dans quelle mesure l'Afrique saura tirer parti de cette conjoncture a priori favorable et comment elle participera à la satisfaction de cette demande supplémentaire. Quant à l'autosuffisance, elle ne pourra être atteinte qu'à condition que des investissements conséquents soient réalisés dans le secteur de la production. 